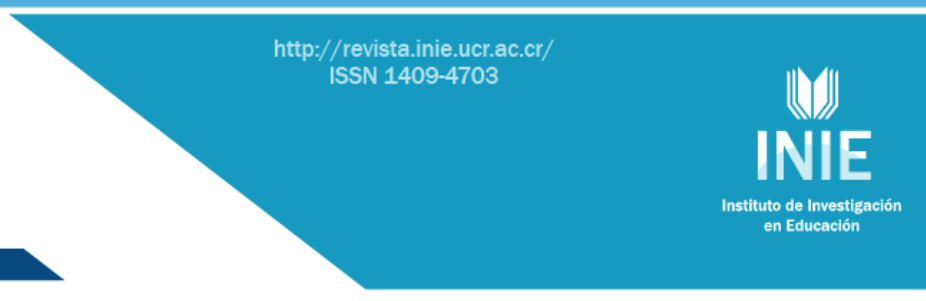

UN ESTUDIO EXPLORATORIO PARA CARACTERIZAR LAS PRÁCTICAS DE LECTURA EN UN GRUPO DE JÓVENES UNIVERSITARIOS Y NO UNIVERSITARIOS

AN EXPLORATORY STUDY BY CARACTERIZATION OF LECTURE PRACTICES IN A GROUP OF UNIVERSITY STUDENTS AND NON- UNIVERSITARIAN YOUNG PEOPLE

\author{
Volumen 13, Número 2 \\ Mayo - Agosto \\ pp. 1-17
}

Este número se publicó el 30 de mayo de 2013

Klency González Hernández

Ana María Castro Laguardia

Revista indizada en REDALYC,

Revista distribuida en las bases de datos:

CATÁLOGO DE LATINDEX, IRESIE, CLASE, DIALNET, DOAJ, E-REVIST@S, SHERPA/ROMEO, QUALIS, MIAR

Revista registrada en los directorios:

ULRICH'S, REDIE, RINACE, OEI, MAESTROTECA, PREAL, CLASCO 


\title{
UN ESTUDIO EXPLORATORIO PARA CARACTERIZAR LAS PRÁCTICAS DE LECTURA EN UN GRUPO DE JÓVENES UNIVERSITARIOS Y NO UNIVERSITARIOS
} AN EXPLORATORY STUDY BY CARACTERIZATION OF LECTURE PRACTICES IN A
GROUP OF UNIVERSITY STUDENTS AND NON- UNIVERSITARIAN YOUNG PEOPLE

\author{
Klency González Hernández ${ }^{1}$ \\ Ana María Castro Laguardia²
}

Resumen: El presente trabajo es un artículo científico que concibe la lectura como una práctica socio-cultural con características y formatos variados. Su objetivo es abordar el estudio de las prácticas de lectura de un grupo de jóvenes universitarios y no universitarios de La Habana, Cuba. Los estudiantes universitarios que integran la muestra pertenecen a las carreras de Psicología y Matemática respectivamente, mientras que los jóvenes restantes no tienen ningún vínculo académico. Para evaluar las prácticas de lectura usamos un cuestionario que consta de 11 preguntas. Los resultados muestran que no existen diferencias significativas entre las prácticas de lecturas llevadas a cabo por los sujetos pertenecientes a ambos grupos, lo cual sugiere que, en este caso, la inserción en el contexto académico no está siendo un factor de significativa importancia en la elección de los libros, las fuentes de información, las vías de acceso, ni en las acciones que se realizan con ellos.

Palabras clave: PRÁCTICAS DE LECTURA, ESTUDIANTES UNIVERSITARIOS, ESTUDIANTES NO UNIVERSITARIOS, CUBA

\begin{abstract}
The present project is a scientific article that conceives reading as a socio-cultural practice with varied features and formats. It aims to address the study of reading practices of a group of young university students and non university students of Havana, Cuba. The university students comprising the sample belong to the careers Psychology and Mathematics respectively. To evaluate reading practices we used a questionnaire consisting of 11 questions. Results show no significant differences between reading practices taken by the students from both groups, which suggests that in this case, the inclusion in an academic context is not being a significant factor in choosing books, information sources, access paths, not even in the actions that are done with them.
\end{abstract}

Keywords: LECTURE PRACTICE, UNIVERSITARY STUDENTS, NON-UNIVERSITARIAN YOUNG PEOPLE, CUBA

\footnotetext{
1 Profesora Auxiliar y Jefa del Departamento de Formación Básica de la Facultad de Psicología de la Universidad de la Habana. Máster en Psicología Educativa. Dirección electrónica: klency@psico.uh.cu

${ }^{2}$ Estudiante de tercer año de la Facultad de Psicología, Universidad de La Habana, Cuba.
}

Artículo recibido: 22 de octubre, 2012

Aprobado: 25 de abril, 2013 


\section{Introducción}

Leer es mucho más que el simple acto de pasar la vista por lo escrito o impreso, haciéndose cargo del valor y significación de los caracteres empleados y pronunciando o no las palabras representadas por estos caracteres. La práctica de la lectura está estrechamente relacionada con aspectos como la representación que hacen los sujetos de la misma, su grado de escolaridad, la sociedad donde viven, sus historias de vida, etc. Visto así, el acto de leer está influenciado, en gran medida, por la cultura.

Diversos autores (Barton y Hamilton, 2002, Peroni, 2003, Petit, 1999) con múltiples fines han convocado la revisión de conceptos muy empleados en las investigaciones acerca del tema, tales como codificación y comprensión lectora, para profundizar en la influencia de lo cultural y romper con la imagen exitosa que se tiene de la lectura cuando realmente los datos cuantitativos dicen lo contrario.

En los contextos educativos, fundamentalmente, crecemos escuchando frases como: la lectura es un camino bello y lleno de aventuras, la lectura te permite viajar a mundos indescriptibles, leer nos provee de múltiples conocimientos, etc. Creer abiertamente en estas metáforas da lugar a ideas erróneas en relación con qué es leer.

Los nuevos estudios de literacidad, campo emergente que desde hace veinte años, ha reunido a estudiosos de múltiples disciplinas para reflexionar acerca la literacidad, como práctica social, que aborda estas cuestiones de forma distinta. La literacidad se entiende como una tecnología que está siempre inmersa en procesos sociales y discursivos, representando la práctica de lo letrado no solo en programas escolares sino en cualquier contexto sociocultural. Incluye tanto la escritura como la lectura, los cuales, aunque son procesos distintos, están íntimamente relacionados y constituyen parte de la experiencia de lo letrado (Barton y Hamilton, 2002).

Desde este enfoque "se estudian las prácticas letradas en ámbitos culturales específicos, pasando de lo universal a lo particular con la ayuda de métodos etnográficos" (Zavala, Niño-Murcia y Ames, 2004 , p. 9). Se trata de observar prácticas de lectura y escritura particulares como íntegramente conectadas al mundo social, a partir de determinadas formas de actuar, de creer, y de diversas maneras de interactuar con el lenguaje oral, utilizando herramientas y tecnologías.

La teoría social de la literacidad enuncia dos unidades básicas: prácticas y eventos letrados. Las prácticas letradas son "formas culturales generalizadas de uso de la lengua 
escrita (...) son lo que la gente hace con la literacidad" (Street, 2003, p. 12). Sin embargo, dichas prácticas no son unidades de comportamiento observables, pues también implican una serie de valores, actitudes, sentimientos y relaciones sociales. Las prácticas letradas, como procesos internos del individuo, son al mismo tiempo procesos sociales que conectan a las personas entre sí, incluyendo conocimientos compartidos representados en ideologías e identidades sociales. Así, las prácticas toman forma a partir de normas sociales que regulan el uso y la distribución de textos, a la vez que prescriben la posibilidad de producirlos y tener acceso a ellos.

Los eventos letrados son actividades en las cuales la literacidad cumple un papel. Son episodios observables que surgen de las prácticas y son formados por estas. La noción de evento acentúa la naturaleza situacional de la literacidad con respecto a que esta siempre existe en un contexto social. El punto de partida para el análisis de la lengua hablada debería ser el evento social de la interacción verbal, antes que las propiedades lingüísticas formales de los textos aislados (Barton y Hamilton, 1998, citado de Zavala, Niño-Murcia y Ames 2004, p. 114).

Teniendo en cuenta el concepto de práctica letrada, más que enfatizar en cuánto tiempo leen los sujetos o con qué velocidad y precisión lo hacen, las investigaciones deberían centrarse en las características que van tomando las prácticas de lectura, indagar en los espacios de dichas prácticas, en las historias de lectura de los individuos, en el acceso que han tenido a los libros, etc. Al parecer, los jóvenes exploran diversos espacios de lectura acercándose a los libros desde la utilidad que para ellos tienen, pero, en la mayoría de los casos, han roto con los estándares clásicos de dichas prácticas, lo que puede significar que no leen los libros de principio a fin, ni declaran algunas lecturas funcionales por no considerarlas legítimas, aún cuando les hayan aportado conocimientos y vivencias.

La primacía de esta noción de "práctica de lectura" en el imaginario social vuelve invisibles una gran cantidad de prácticas de lectura de estos jóvenes que siguen "dominados" por la representaciones literarias de lectura aprendidas en la escuela primaria (Lahire, 2004).

Este autor sistematiza cuatro criterios en relación con los cuales los sujetos suelen evaluar una lectura como legítima. El primero, tiene que ver con que son lecturas que se realizan para sí mismos (no se incluyen por tanto en esta categoría libros o fragmentos leídos a otras personas, por ejemplo cuentos para niños). El segundo, son lecturas que no 
resultan utilitarias, es decir, aquellas que no se llevan a cabo como medio para desarrollar otra actividad como la confección de trabajos escolares. El tercero, se realizan íntegramente, debe ser una lectura completa de un libro, de principio a fin, las lecturas fragmentadas no se consideran. El último, deben ser textos considerados legítimos por sí mismos, es decir, aquellos con autor reconocido, recordable y que sea relativamente extenso, por tanto, en raras ocasiones se suele declarar la lectura de comics, fotonovelas o novelas policíacas.

Una idea similar es descrita por Donnat (2004), quien distingue entre prácticas reales y prácticas declaradas. Lo que los lectores expresan puede estar sobreestimado, pero también subestimado, porque las prácticas de lectura han perdido en la actualidad su poder de distinción. El libro y la lectura se han vulgarizado. En muchas ocasiones se tienden a olvidar las lecturas utilitarias o no se registran como tales. Aparece aquí el tema de la legitimidad: si bien una persona tiende a sobreestimar sus propias prácticas para dar una imagen positiva de sí misma, tiene también a subestimarlas al no declarar sino lo que considera legítimo para una situación de encuesta.

Por ejemplo, no se reportan las lecturas fragmentadas y funcionales, porque el ideal de lectura es la lectura lineal, que consume tiempo y exige empeño y que va del inicio hasta el fin del libro. Entonces, el indicador "número de libros leídos" resulta bastante relativo para utilizarlo como la medida del continuo entre lectores asiduos a no lectores.

En una investigación realizada por Chartier (2004) se confirman estas ideas. Los resultados muestran que los sujetos, cuando tienen que reportar libros leídos, nombran "los libros leídos de manera lineal, desde el principio al fin" (Chartier, 2004, p. 115). A pesar de que la consigna especifique marcar todos los libros hojeados o consultados parcialmente, estos son eliminados del reporte del entrevistado. Tratando de recordar un máximo de títulos, los estudiantes toman conciencia de que practican una lectura fragmentada (cursos, fotocopias de capítulos, artículos de diarios, etc.). El cruce de estas variadas lecturas, que interrumpen la lectura continuada de obras más largas, aparece como un obstáculo suplementario, que impide la reconstrucción integral y fiable de los recorridos de lectura.

Bahloul (2003) hace referencia a las aristas que caracterizan las prácticas de lectura, describiendo lo que él denomina escenarios de poca lectura. Plantea que esta clase de práctica corresponde con el reducido volumen de información que posee el lector sobre los libros y con una tendencia a la no planeación ni estructuración de la elección y de la lectura, 
lo cual conlleva a que, dadas sus condiciones de préstamo, no se considere la biblioteca como un espacio de información ni de adquisición de obras.

La lectura revela en sus diferentes movilidades estar estrechamente asociada al contexto en el que surge y cobra sentido: por ello, no es posible adquirirla o perderla de manera definitiva. Además, a pesar de que suele ser considerada uno de los actos más individuales que existen, no deja de ser una operación de construcción social de la realidad. Con esta expresión designamos la doble interacción, por una parte, entre lo que yo leo (lo que puedo leer, lo que tengo ganas de leer, etc.) y lo que considero real en el mundo y, por otra, entre el sentido que le doy al acto de lectura y la definición de la situación en la que se inscribe (Peroni, 2003).

Desde este punto de vista no podríamos decir que una experiencia lectora sea menor que otra. Más oportuno sería hablar de lectura estrecha para designar una práctica regida por un imperativo de adecuación del mundo del texto a una realidad; oponiéndose, en este sentido, a una lectura abierta que considera, con base en el modelo letrado, el mundo del texto por sí mismo.

La lectura es percibida por algunos individuos como un ocio y como una huida del ritmo de vida profesional y activa, apareciendo como lo negativo del empleo del tiempo estructurado y planificado de la vida activa. Se piensa en leer cuando no hay otras actividades que realizar como mirar la TV, interactuar con las nuevas tecnologías, etc. Al situarla en el orden de lo pasivo, la "poca lectura" no es sistematizada y rehuye toda socialización planificada referente al libro. Más que en dimensión cuantitativa, aparece como el efecto cualitativo de la marginación del libro en relación con la vida activa programada. La lectura está dentro de lo pasivo, por ello, no pueden sino concedérsele los llamados tiempos libres que surgen fuera de lo programado en la vida activa (Peroni, 2003). Este mismo hecho influye en el tipo de obras que suelen recordarse cuando se pide a los lectores enunciar obras leídas en el pasado.

Teniendo en cuenta la influencia de la cultura en el acto de leer, la necesidad de realizar nuevas investigaciones con la perspectiva de los nuevos estudios de literacidad para observar prácticas de lectura particulares, y la importancia de describir e indagar en los espacios de dichas prácticas, nuestra investigación se propone caracterizar las prácticas de lectura en un grupo de jóvenes universitarios y no universitarios cubanos. 
Con ese objetivo esperamos explorar en el grupo de estudio algunas distinciones de la lectura como práctica cultural, identificando la influencia del contexto educacional en las prácticas, los espacios privilegiados, las principales vías de socialización de la lectura, así como elaborar hipótesis sobre las representaciones sociales de la lectura que poseen los sujetos estudiados. Todo esto, tomando en cuenta la necesidad de enfocarse en las verdaderas formas de rescate de las prácticas de lectura para poder, en investigaciones posteriores, proponer programas de promoción lectora acordes con las verdaderas intenciones de los sujetos que las practiquen.

\section{Metodología}

El grupo de estudio está integrado por 60 jóvenes residentes en La Habana, Cuba y que tienen entre 19 y 21 años de edad. De ellos, 30 pertenecen al sexo femenino y 30 al sexo masculino. En cuanto al nivel educacional tenemos que 40 son estudiantes de La Universidad La Habana, 20 pertenecen a la Facultad de Psicología y 20 a la Facultad de Matemática y Computación. Los restantes 20 jóvenes no tienen ningún vínculo académico. La Tabla $\mathrm{N} . \stackrel{0}{1}$ ilustra mejor estos datos.

Tabla 1: Características de los jóvenes estudiados

\begin{tabular}{|l|c|c|c|c|}
\hline \multicolumn{3}{|c|}{ Estudiantes universitarios } \\
\hline & \multicolumn{2}{|c|}{ Facultad Psicología } & \multicolumn{2}{|c|}{ Facultad Matemática } \\
\hline Promedio de edad & \multicolumn{2}{|c|}{19,7 años } & \multicolumn{2}{c|}{20 años } \\
\hline Cantidad por sexo & $\begin{array}{c}10 \\
\text { mujeres }\end{array}$ & $\begin{array}{c}10 \\
\text { hombres } \\
\text { mujeres }\end{array}$ & $\begin{array}{c}10 \\
\text { hombres }\end{array}$ \\
\hline Total & \multicolumn{2}{|c|}{20} & \multicolumn{2}{|c|}{20} \\
\hline & Jóvenes no universitarios \\
\hline Promedio de edad & \multicolumn{3}{|c|}{21,2 años } \\
\hline Cantidad por sexo & \multicolumn{3}{|c|}{10 mujeres } \\
\hline Total & \multicolumn{3}{|c|}{20 hombres } \\
\hline
\end{tabular}


Utilizamos el cuestionario elaborado por Chartier (2004), que consta de diez ítems y permite indagar acerca de las peculiaridades de la práctica de la lectura en los grupos estudiados, entre ellas: el tipo de literatura consumida (o al menos declarada), las fuentes de información acerca de los libros, las vías de obtención de estos, las acciones que se realizan durante el consumo literario, las formas de socialización de lo leído, etc. (ver Anexo 1 para una revisión del cuestionario).

Para caracterizar las prácticas de lectura que llevan a cabo los estudiantes universitarios y no universitarios, se han calculado los promedios de selección de cada uno de los ítems, comparando los resultados con los declarados por Chartier (2004).

El carácter exploratorio de la presente investigación permite solo una aproximación al problema en cuestión y, dada la naturaleza de los grupos de estudio, no resulta posible realizar una generalización de los resultados a otros grupos con características sociodemográficas semejantes. Sin embargo, en investigaciones futuras podemos implementar programas de promoción de la lectura teniendo en cuenta la caracterización que de las prácticas logremos con este estudio.

\section{Resultados y discusión}

A continuación expondremos los resultados en el orden siguiente: primero mostraremos los elementos más significativos en torno a las prácticas de lectura encontrados en cada uno de los grupos, a partir de los ítems del cuestionario. Posteriormente, se procederá a un análisis comparativo de los resultados obtenidos.

\subsection{Resultados del grupo de estudiantes universitarios}

Del total de estudiantes universitarios encuestados, el 90\% propone el título de alguna obra. En cuanto a los autores de estos libros, el 85\% de los estudiantes hace mención a estos y Paulo Coelho es el autor más señalado con tres títulos diferentes: Once minutos, Verónica decide morir y A orillas del Río Piedra me senté y lloré. Por otra parte, solo el 25\% conoce la editorial y el $10 \%$ la colección del libro escogido.

En este sentido, es esencial tomar en cuenta las características del contexto sociocultural en el que se realiza el estudio. En nuestro país, las editoriales son todas de carácter estatal, existiendo espacios limitados para promocionar y publicitar sus ofertas, así como falta de competencia entre ellas, lo que pueden ser factores que sesguen la atención de los 
sujetos al comprar un libro. Es decir, en muchas ocasiones los criterios que llevan a los sujetos a seleccionar libros no incluyen la editorial a la que estos pertenecen. Por su parte, en el sistema de enseñanza cubano no se realiza un especial énfasis en la relevancia de las editoriales en relación con la calidad de las obras literarias. Podría afirmarse, entonces, que como tendencia, el conocimiento de la editorial o colección no constituye un factor de selección del libro para los sujetos que componen este grupo.

Teniendo en cuenta las acciones realizadas con el material literario, la totalidad de los jóvenes encuestados asegura haberlo leído; un 15\% asegura, además, haberlo recorrido, (lo que significa hojearlo, leerlo por partes, detenerse en algunos fragmentos, comentarlo, etc.), un $5 \%$ lo ha leído parcialmente en alguna ocasión y un $20 \%$ lo ha consultado. Estos datos resultan congruentes con los resultados hallados por Lahire (2004) y Chartier (2004), por lo que puede aventurarse que los sujetos que conforman este grupo de estudio tienden a considerar como lecturas declarables aquellas que han consumido íntegramente, aunque puedan haber realizado en otros momentos acciones que impliquen una consulta o lectura parcial.

Asimismo, el hecho de que un pequeño porcentaje de los sujetos del grupo señale haber consultado el libro como medio para la realización de alguna otra tarea o deber laboral apunta a que el grupo tienda a no considerar como legítima, empleando un término lahiriano, aquella lectura que resulta utilitaria. Lo mismo se reafirma con los resultados obtenidos para el ítem 10 del cuestionario, relacionado con la utilización del libro declarado en el ámbito laboral o, en este caso, escolar de los sujetos. El 25\% utilizó el libro en alguno de estos ámbitos; de ellos, el 15\% lo empleó para hacer un resumen, una ficha de lectura o una presentación, el 10\% para realizar deberes del trabajo, mientras que solo un 5\% lo requirió para preparación pedagógica.

En cuanto a la relación de los miembros del grupo con los libros, obtuvimos que un $85 \%$ de los estudiantes había oído hablar del libro anteriormente; de ellos un 15\% obtuvo información de él a través de los medios de comunicación, otro 15\% por medio de un profesor o representante institucional; mientras que un $20 \%$ lo conoció mediante un colega, un $65 \%$ a través de un amigo y un $20 \%$ mediante otro libro. Ahora, haciendo referencia a la forma en que el libro llegó a las manos de estos estudiantes, encontramos que un $20 \%$ lo adquirió mediante la compra, para un $25 \%$ fue un regalo, mientras que un $15 \%$ lo obtuvo mediante un préstamo institucional, un $25 \%$ a través de un préstamo social y, por último, un 
$15 \%$ de otra forma no especificada. Estos resultados no distan mucho de los obtenidos por Bahloul (2003), y apuntan a que en nuestro contexto los espacios institucionales, específicamente las bibliotecas, no constituyen fuentes privilegiadas para la obtención de los libros que los lectores declaran consumir.

Explorando la cantidad de tiempo dedicado a la lectura encontramos que estos estudiantes reportan dedicar un promedio de 3 horas diarias a la lectura del libro. Respecto a la pregunta sobre qué hicieron con el libro, hallamos que un 45\% de los estudiantes lo leyó, otro 35\% lo leyó marcando pasajes, mientras que un 20\% lo leyó y tomó notas, y solo un 5\% lo fotocopió. El 100\% de los sujetos refiere haber hablado sobre el libro con alguien más, un $60 \%$ lo hizo con alguna persona de su familia, el $100 \%$ con sus amigos, otro $45 \%$ con sus colegas, mientras que un $20 \%$ lo mencionó con otras personas.

Como otro aspecto de interés, encontramos que el libro escogido fue recomendado a otra persona por un $90 \%$ de los estudiantes, de ellos un 35\% lo recomendó a familiares, un $85 \%$ a amigos, otro $40 \%$ a colegas, mientras que un $5 \%$ lo recomendó a otras personas. Otro dato importante recogido en las encuestas es el hecho de que el 60\% de los estudiantes le prestó o regaló el libro a alguien más; de ellos un 40\% fue a familiares, un $45 \%$ a amigos, un $15 \%$ a colegas y solo un $5 \%$ fue a otras personas. Por último, hallamos que el tipo de obra seleccionada por los sujetos a quienes se les aplicó el cuestionario fue en un $80 \%$, de carácter literario, en un $10 \%$ informativo o no literario y, para terminar, un $10 \%$ de los estudiantes no sabía de qué tipo de obra se trataba el libro escogido.

Al parecer, las prácticas de lectura de este grupo de jóvenes incluyen comentarios de sus lecturas lo que puede ser motivador para que otras personas a su alrededor lean el libro. Cuando la lectura se recomienda entre personas cercanas se va creando una atmósfera positiva favorecedora de la misma actividad y del intercambio de opiniones sobre los temas tratados en el libro, así como su relación con la vida cotidiana de los sujetos. En este sentido, ese tipo de lectura se vuelve legítima y en eventos futuros probablemente los sujetos las declararan.

\subsection{Resultados del grupo de jóvenes no universitarios}

El 100\% del grupo formado por jóvenes sin vínculo académico conoce el título del libro al que hace referencia, mientras que solamente un $80 \%$ refiere el autor. Únicamente un 30\% conoce la editorial y un $20 \%$ la colección. En este grupo, el conocimiento sobre algunos 
datos del libro que pueden brindar información sobre su procedencia, su calidad, etc., son desconocidos por los sujetos. Al igual que en el grupo anterior esto puede tener que ver con el contexto cubano donde no se pondera el conocimiento ni de la editorial, ni de las colecciones.

En relación con la lectura que realizaron obtuvimos que el 75\% de los sujetos leyó el libro de forma íntegra, un 5\% se limitó a recorrerlo y un 10\% abandonó la lectura una vez comenzada. Un 15\% lo leyó parcialmente, este es el mismo porcentaje de los que lo han utilizado como consulta. Estos resultados concuerdan con los encontrados por Chartier (2004) que dicen que los lectores tienden a recordar y por ende a declarar aquellas obras que hayan leído de principio a fin. Aparece la distinción entre lectura legítima (que parece ser finalmente la que se recuerda y se declara y que se define por: lectura autónoma, para uno mismo, de un texto legítimo, relativamente largo, leído de principio a fin y atribuible a un autor) y lecturas entrecortadas, discontinuas, informativas, rápidas, técnicas, documentales, etc. (Lahire, 2004).

Un $75 \%$ de los jóvenes había escuchado del libro con anterioridad a su lectura, de los cuales el $10 \%$ tuvo conocimiento a través los medios de comunicación (revista, radio, televisión), un 15\% mediante un profesor o representante institucional, el 10\% por un colega, $20 \%$ por amigos y solo un $5 \%$ por otros medios no señalado en el cuestionario. Ningún sujeto del grupo tuvo conocimiento del libro a través de su cita en otro libro.

Sobre cómo tuvieron acceso al libro al que hacen referencia, un $80 \%$ del grupo lo compró, el $25 \%$ lo obtuvo como obsequio, el $10 \%$ tuvo acceso a través del préstamo institucional y un 50\% mediante préstamo social (amigo, familia, colega). En este ítem queremos destacar dos elementos. El primero tiene que ver con que el porcentaje de jóvenes que declara haber comprado el libro es alto, y eso al parecer caracteriza estas prácticas de lectura y tiene que ver con que los precios de los libros en el mercado cubano son bajos, todo el que quiera acercarse a la lectura de libros de calidad puede comenzar a hacer su propia biblioteca, pues los libros tienen precios muy accesibles a la población. En segundo lugar, el número de jóvenes que adquiere el libro mediante préstamo institucional es muy bajo, lo que apunta a un detrimento de las bibliotecas como fuentes de obtención de libros y, por tanto, de conocimientos. En palabras de Bahloul, "los lugares en los que el presunto lector adquiere el libro brindan valiosa información sobre el conocimiento que posee del mismo, adquisición y conocimiento del libro son indisociables” (Bahloul, 2003, p. 56). 
En cuanto a la pregunta referida a qué hicieron con el libro, encontramos que el $70 \%$ de los jóvenes simplemente se limitó a leerlo, un 20\% lo leyó marcando los pasajes, las páginas, anotando los márgenes; el 15\% lo leyó y tomó notas; mientras que nadie lo fotocopió. De manera espontánea tuvo la oportunidad de referirse al libro el $85 \%$ de los sujetos, de estos el $45 \%$ lo hizo con su familia, el $65 \%$ con amigos, el $15 \%$ con colegas y un $5 \%$ con otros no señalados en el cuestionario. El libro fue recomendado por un $75 \%$ de los sujetos, siendo un $30 \%$ a la familia, el $45 \%$ a un amigo y el $25 \%$ a colegas. La cantidad de jóvenes que comentan o recomiendan el libro a sus amigos es elevado, y esto tiene que ver con la edad de desarrollo en que se encuentran, donde los amigos son un grupo de referencia y de apoyo con mucho valor.

Exclusivamente, el 15\% utilizó el libro referido en su trabajo. De estos, el 10\% fue para realizar un deber o una tarea, mientras que un 5\% para una preparación pedagógica. Un alto porcentaje de los jóvenes no universitarios refieren haber consumido literatura recreativa. El $75 \%$ de los libros referidos por los sujetos de este grupo fueron clasificados dentro del género literario; el 10\% como libros informativos o no literarios y un 15\% desconocía la clasificación del libro al que se refirió en el cuestionario. En este sentido, se aprecia una tendencia a declarar el consumo de literatura que no resulte utilitaria, es decir, aquella que se consume solamente con fines recreativos.

En general, los libros que circulan y se intercambian son ante todo lecturas personales, aunque se aprecian lecturas compartidas, entre ellas títulos clásicos, pertenecientes a la creación de García Márquez como Cien años de soledad y El amor en los tiempos del cólera, y otros que caben en la categoría de best seller de moda como: Harry Potter (diversos títulos de la saga), El código Da Vinci, Once minutos, Verónica decide morir y $A$ orillas del Río Piedra me senté y lloré, estos tres últimos de la autoría de Pablo Coelho. En este sentido, cabe destacar que esta condición de best seller parece ser considerada por los sujetos de ambos grupos como un criterio de selección de los títulos. Incluso en Cuba, donde la publicidad en los medios de comunicación masiva que se hace de esos títulos es mínima, al parecer por medios colaterales como la Internet, o el préstamo de libros, los sujetos tienen acceso a ellos y los leen y luego los declaran considerando su lectura legítima y digna de ser socializada. 


\subsection{Comparación de los resultados obtenidos en ambos grupos}

Llama la atención el hecho de que no existen diferencias significativas entre las lecturas declaradas por los sujetos de ambos grupos: estudiantes universitarios y no universitarios. Tampoco existen diferencias significativas entre las lecturas declaradas por los estudiantes universitarios provenientes de las carreras de Psicología y Matemática (aunque en el caso de estos últimos, se registra que un $10 \%$ refiere títulos específicamente relacionados con sus estudios universitarios).

Este hecho lo podemos explicar hipotetizando que la representación social de los jóvenes sobre la lectura se sustenta en considerarla una práctica recreativa. Por tanto, no tienden a declarar las lecturas realizadas para cumplir propósitos académicos, emergiendo el hecho que tiendan a recordarse y declararse como tendencia aquellas lecturas que se han realizado íntegramente (por lo general las lecturas académicas se realizan de forma fragmentada), en un momento reciente o que constituyen best seller.

Al parecer, estamos en presencia en estos jóvenes de una contradicción entre prácticas reales y prácticas declaradas (Donnat, 2004). Lo que la mayoría de los lectores declaran, especialmente los estudiantes universitarios, está subestimado. Solo el $10 \%$ de la muestra de estudiantes de la carrera de Matemática expresa leer libros relacionados con su perfil profesional. Los estudiantes de Psicología no declaran como legítimos los fragmentos de libros leídos para estudiar un seminario, o salir bien en un examen. Hay un olvido de las lecturas utilitarias, ponderando aquellas lecturas lineales, que consumen tiempo y exigen empeño y que van del inicio hasta el fin del libro.

Las lecturas que tienden a ser compartidas por un alto número de estudiantes universitarios y no universitarios pertenecen a autores como García Márquez y Pablo Coelho. Encontramos, también, con una alta frecuencia, títulos recomendados para un período etáreo inferior al que se encuentran estos sujetos, como el caso de la saga de Harry Potter. Estos últimos títulos se venden en nuestro país en muy escasas librerías y a muy altos precios, lo cual hace pensar en que en la mayoría de los casos son consumidos en formato digital, aunque esto es un asunto que abre nuevos caminos de investigación.

En ambos grupos se observa una marcada tendencia a no considerar las bibliotecas e instituciones educativas como fuentes de información y obtención de obras. Nótese el bajo porcentaje de sujetos que refieren haber obtenido el libro reseñado a través de ellas (15\% y $10 \%$ respectivamente). Esto se une al hecho de que la mayoría de los sujetos refiere como 
fuente de información privilegiada los medios de comunicación, lo cual resulta contradictorio, porque en Cuba generalmente la publicidad que se realiza en los medios de comunicación se relaciona con campañas de promoción de la lectura y con espacios particulares de venta de libros, pero casi nunca se refiere a la comercialización de best seller y libros "de moda", lo cual nos hace pensar que la información de que disponen los jóvenes acerca del libro antes de ser leído resulta superficial, y se basa en criterios externos como su popularidad.

Por su parte, la familia y los grupos informales constituyen para la mayoría de los jóvenes estudiados, los contextos fundamentales para la socialización de la lectura. Esto puede resultar paradójico en el caso de los estudiantes universitarios, ya que su inserción en este ámbito académico con determinadas características y exigencias debería promover en sí misma la socialización y práctica de la lectura. La deficiente ocurrencia de este fenómeno se refuerza con el hecho de que únicamente el $25 \%$ de los estudiantes encuestados refiere haber tenido la oportunidad de emplear el libro en cuestión en la realización de labores escolares-profesionales. Lo anterior constituye un criterio señalado por Bahloul (2003) para identificar los escenarios de poca lectura. Este fenómeno también puede estar apuntando a que la opinión social de los grupos informales en los que se desarrollan estos sujetos, pueda estar condicionando el tipo de literatura que consumen o al menos, que declaran consumir.

Al estar la lectura totalmente imbricada en la organización y las condiciones sociales, su iniciativa, la recepción y circulación de los conocimientos adquiridos, las representaciones del libro y de lo que se lee actúan en el marco de las "redes" de socialización. De esta forma, deberemos potenciar desde la educación primaria escenarios donde los individuos inicien o mantengan sus prácticas lectoras (Bahloul, 2003). Asimismo, habría que justificar que edad es más apropiada para que el niño comience a adquirir estrategias de lectura que le permitan relacionar lo que lee con sus conocimientos previos y autocuestionarse la lectura y su vida en función también de lo leído. Como la lectura es evolutiva (Peroni, 2003), siendo jóvenes sería importante insertarlos en múltiples y ricas prácticas que mantengan o aumenten una apropiación enriquecedora de valores, conocimientos y vivencias de nuestra cultura. Así como familiarizarlos con visiones distintas de lecturas donde reconozcan y declaren las prácticas funcionales como legítimas y útiles en su vida cotidiana. 


\section{A modo de conclusiones}

No existen diferencias significativas entre las prácticas de lecturas llevadas a cabo por los sujetos pertenecientes a ambos grupos, lo cual sugiere que, en este caso, la inserción en el contexto académico no está siendo un factor de significativa importancia en la elección de los libros, las fuentes de información, las vías de acceso, ni en las acciones que se realizan con ellos.

Existe contradicción entre prácticas reales y prácticas declaradas. Los jóvenes estudiados solo declaran los libros que leen de principio a fin, refiriendo títulos que para ellos tienen un valor agregado, lo que se convierte en la mayoría de los casos en libros de moda, o libros de autores muy conocidos.

Hay una tendencia a no considerar los espacios institucionales como fuentes de información y obtención de libros, lo que constituye un llamado de atención a quienes están a cargo de desarrollar políticas que fomenten el consumo literario, pues dichos centros poseen un amplio caudal de obras de alto valor cultural $y$, asimismo, tienen la potencialidad de constituir vías de socialización de la lectura por excelencia.

En futuras investigaciones relacionadas con el tema en el contexto cubano, se propone emplear métodos y técnicas que permitan el abordaje de las historias de vida relacionadas con la lectura de los sujetos de estudio, así como las representaciones sociales que estén condicionando sus prácticas.

Consideramos que sería conveniente trabajar sobre los mitos existentes alrededor de la lectura para desmontar algunos de ellos y que las personas lean, a su manera, los libros que deseen, y se sientan en total libertad de defender su elección sin sentirse evaluado.

\section{Referencias}

Bahloul, Joëlle. (2003). Lecturas precarias. Estudio sociológico sobre los pocos lectores. México: SEP/FCE.

Barton, David y Hamilton, Mary. (2002). La literacidad entendida como práctica social. En Virginia Zavala, Mercedes Niño-Murcia y Patricia Ames (eds), Escritura y sociedad. Nuevas perspectivas teóricas y etnográficas (pp. 109-139). Lima: Red para el desarrollo de las ciencias sociales en el Perú.

Chartier, Anne Marie. (2004). La memoria y el olvido, o cómo leen los jóvenes profesores. En Bernard Lahire, Sociología de la lectura (pp.109-137). España: Gedisa, Colección LEA. 
Donnat, Olivier. (2004). Encuestas sobre los comportamientos de lectura. Cuestiones de método. En Bernard Lahire (comp.), Sociología de la lectura (pp. 59-84). Barcelona: Gedisa.

Lahire, Bernard. (2004). Formas de lectura estudiantil y categorías escolares de la comprensión de la lectura. En: Bernard Lahire (comp.), Sociología de la lectura (pp.149-178). Barcelona: Gedisa.

Petit, Michelle. (1999). Nuevos acercamientos a la lectura y los jóvenes. México: Colección Espacios para la lectura. FCE.

Street, Brian. (2003). Los nuevos estudios de literacidad. En Virginia Zavala, Mercedes NiñoMurcia y Patricia Ames (eds), Escritura y sociedad. Nuevas perspectivas teóricas y etnográficas (pp. 81-107). Lima: Red para el desarrollo de las ciencias sociales en el Perú.

Peroni, Michel. (2003). Historias de lectura trayectorias de vida y lectura. México: SEP/ Fondo de Cultura Económica.

Zavala, Virginia, Niño-Murcia, Mercedes, Ames, Patricia. (2004). Escritura y sociedad. Nuevas perspectivas teóricas y etnográficas. Lima: Red para el desarrollo de las ciencias sociales en el Perú. 


\section{Anexos.}

\section{1- Anexo 1: Cuestionario sobre prácticas de lectura (Chartier, A. M., 2004)}

Seudónimo:

Fecha:

1. Indique del libro en cuestión:

- título

- autor

-editor

-colección

2. ¿Qué ha hecho usted con el libro? (Marque lo que corresponda)

_ lo ha leído

_ lo ha abandonado

_ lo ha consultado
_ lo ha recorrido

_ lo ha leído parcialmente

3. ¿Usted había oído hablar de este libro antes? Sí

No

Si la respuesta es afirmativa, marque dónde

_ medios de comunicación (revista, radio, televisión)

_ profesor u otro representante institucional

_ colega

_ amigo

_ otro libro leído

- otros

4. ¿Cómo llegó el libro a sus manos?

_ compra

_regalo

_préstamo institucional

_ préstamo social (familia, amigo, colega, oto)

- otros

5. ¿Cuánto tiempo pasó en contacto con el libro? cantidad aproximada de horas

repartidas en cuánto tiempo 
6. ¿Qué hizo con el libro?

_ simplemente lo leyó

_ lo leyó marcando pasajes, marcando páginas, anotando los márgenes

_ lo leyó y tomó notas

_lo fotocopió

7. ¿Usted tuvo la ocasión de hablar del libro de manera espontánea? Sí

No

Si la respuesta es afirmativa ¿con quién?

_ familia

_ amigo

_ colega

- otro

8. ¿Usted tuvo la ocasión de utilizar el libro en su trabajo? Sí

No

Si la respuesta es afirmativa ¿para qué?

_ resumen, ficha de lectura, presentación

_ para un deber, un trabajo

_ para una preparación pedagógica

9. ¿Recomendó el libro a alguien? Sí

No

Si la respuesta es afirmativa ¿a quién?

_ familia

_ amigos

_ colegas

_otros

10. ¿Le prestó/regaló el libro a alguien? Sí

No

Si la respuesta es afirmativa ¿con quién?
_ familia
_amigos
_ colegas
_otros 